\title{
Perceived Family Impact of Preschool Anxiety Disorders
}

\author{
Dr. Nissa R. Towe-Goodman, Ph.D., \\ Center for Developmental Science, University of North Carolina-Chapel Hill
}

Dr. Lauren Franz, MBChB,

Duke University Medical Center

Dr. William Copeland, Ph.D., Duke University Medical Center

Dr. Adrian Angold, MRCPsych, and

Duke University Medical Center

Dr. Helen Egger, M.D.

Duke University Medical Center

\section{Abstract}

Objective-We examined the perceived impact of child anxiety disorders on family functioning, because such impact is a key predictor of mental health service receipt. Additionally, we examined the relative impact of preschool anxiety compared to other early childhood disorders, and whether this impact persisted after accounting for the effects of comorbidity, or varied by child age and sex.

Method-Drawing from a pediatric primary-care clinic and oversampling for children at risk for anxiety, 917 parents of preschoolers (ages 2-5 years) completed a diagnostic interview and reported on child psychiatric symptom impact on family finances, relationships, activities, and well-being.

Results-After accounting for comorbid disorders, families of children with anxiety were 3.5 times more likely to report negative impact of their child's behavior on the family relative to nondisordered children. Generalized and separation anxiety had a similar impact on family functioning as ADHD and disruptive disorders. There was a significant family impact for girls with social phobia, whereas there was no impact for boys.

Conclusions-Preschool anxiety has a significant, unique impact on family functioning, particularly parental adjustment, highlighting the family impairment linked with early anxiety, and the need for further research on barriers to care for these disorders.

\footnotetext{
(C) 2014 American Academy of Child \& Adolescent Psychiatry. Published by Elsevier Inc. All rights reserved.

Correspondence to: Nissa Towe-Goodman (ntowe@live.unc.edu), Center for Developmental Science, The University of North Carolina at Chapel Hill, 100 E. Franklin St., Suite 200, Chapel Hill, NC, 27599-8115, USA; Phone: (828) 231-9199; Fax: (919) 966-4520.

The content is solely the responsibility of the authors and does not necessarily represent the official views of the National Institutes of Health. The authors would like to thank Ben Goodman, PhD, of Duke University Center for Child and Family Policy, and the three anonymous reviewers for their helpful comments.

Publisher's Disclaimer: This is a PDF file of an unedited manuscript that has been accepted for publication. As a service to our customers we are providing this early version of the manuscript. The manuscript will undergo copyediting, typesetting, and review of the resulting proof before it is published in its final citable form. Please note that during the production process errors may be discovered which could affect the content, and all legal disclaimers that apply to the journal pertain.
} 


\section{Keywords}

preschool; family impact; generalized anxiety; separation anxiety; social phobia

Anxiety disorders are among the most common psychiatric problems in childhood. ${ }^{1}$ Although there is limited epidemiological work with young children, existing evidence suggests these disorders are present from a very early age, with 6-10\% of preschoolers (children 2-5 years of age) in U.S. community samples meeting diagnostic criteria for an anxiety disorder. ${ }^{2}$ Children with anxiety disorders experience symptoms that are highly distressing, with adverse implications for long-term functioning and development. ${ }^{2,3}$ Although early onset anxiety is associated with significant impairment and may follow a pernicious course without intervention, ${ }^{3}$ it is among the least treated disorders of childhood. ${ }^{4}$ It has been hypothesized that parents may less likely to pursue mental health services for children with anxiety disorders because they have less impact on the family than disruptive behavior problems or attention-deficit hyperactivity disorder (ADHD) ${ }^{4}$ However, there is little research to date on the perceived impact of childhood anxiety disorders on family functioning, particularly in early childhood.

In fact, the phenomenology of preschool anxiety disorders has only recently been explored. ${ }^{2}$ Creating developmentally sensitive diagnostic criteria was an essential step in this process, ${ }^{5}$ such as the Research Diagnostic Criteria-Preschool Age (RDC-PA) ${ }^{6}$ and the Diagnostic Classification of Mental Health and Developmental Disorders of Infancy and Early Childhood: Revised Edition (DC:03-R) ${ }^{7}$ (for a review of these and other assessments of preschool psychopathology, $\mathrm{se}^{8}$ ). Although validation of specific anxiety disorders in young children is ongoing, there is evidence for similar genetic influences, ${ }^{9}$ environmental risk factors, ${ }^{10}$ and patterns of homotypic and heterotypic continuity as found in older children. ${ }^{9,11}$ Similar patterns of differentiation in preschool symptoms are also found, with generalized anxiety disorder (GAD), separation anxiety disorder (SAD), and social phobia (SP) consistently emerging as relatively common, separable syndromes across both younger and older preschoolers. ${ }^{12,13}$. There are some developmental differences in the presentation of symptoms (e.g., for GAD, only one of six symptoms required for diagnosis in childhood; somatic symptoms are relevant across preschool, but worrying may be more salient for 2-3 year olds, while concentration difficulties are more important for older children). ${ }^{2,13}$ Because young children may lack cognitive, verbal, and emotional capacities to describe their anxiety, symptom identification often depends on behavior, using adult-report or observational assessments. ${ }^{2}$ Additionally, because normative anxiety and fears peak within the toddler period, it has been recommended to require impairment for diagnosis. ${ }^{17}$ Although there is increasing recognition that preschoolers experience distressing anxiety that impairs their functioning and development, the perceived family impact of early anxiety is unknown.

Research with school-aged children suggests that having a child with a psychiatric condition negatively impacts family functioning in multiple ways, including increased worries and concerns about the child, additional expenses and loss of income, strained family relationships, impaired social interactions and restricted activities, and decreased parental adjustment. ${ }^{14,15}$ In turn, the perceived family impact of the child's disorder predicts whether or not the child receives needed services, above and beyond diagnosis and level of functioning. ${ }^{14}$ Indeed, research with 6-18-year-olds suggests that the impact of anxiety on caregivers is a key predictor of service receipt, ${ }^{16}$ although only $72 \%$ of school-aged children with impairing anxiety disorders receive any kind of counseling, and younger children are even less likely to receive services. ${ }^{4}$ Additionally, it has been suggested that the perceived family impact of the child's disorder is a critical index of impairment because of the 
embedded nature of young children's behavior within the family context. ${ }^{2}$ Because preschool is not mandatory, the family may be the only setting in which impairment is apparent, and caregivers may alter the environment to minimize effects of the disorder. For example, parents may limit exposure to anxiety provoking situations (e.g., not placing the child in daycare) or change family routines to minimize the child's distress (e.g., the child sleeps in the parents' bed), such that individual markers of impaired functioning are obscured, despite costs to the overall family functioning. ${ }^{2}$

Although research with school-aged children suggests that internalizing problems (i.e., anxiety and depression) may have less impact on the family than externalizing problems, ${ }^{14}$ the relative impact of anxiety may be greater in early childhood. Compared with older children, preschoolers have less independence from caregivers and spend more time at home, making symptoms particularly salient to family members. Although the relative impact of preschool anxiety disorders is unknown, research with 5-15-year-olds suggests that the impact of emotional symptoms on the family is greater in younger children. ${ }^{15}$

Perceived family impact of anxiety disorders may also depend on the child's specific diagnosis and comorbidity. No studies to date have examined the impact of GAD, SAD, or $\mathrm{SP}$, but there is evidence for discrepancies in services receipt; school-aged children with GAD and SP are less likely to receive treatment than those with SAD. ${ }^{8}$ Additionally, although comorbidity between child anxiety and other disorders is documented within the preschool period, ${ }^{2,9}$ it is unclear whether the perceived impact of anxiety persists above and beyond the influence of other disorders. Evidence with 4-12-year-olds suggests internalizing problems have a unique impact on caregiver stress after accounting for externalizing behavior. ${ }^{17}$

Finally, there is reason to believe that the perceived family impact of preschool anxiety may differ for girls and boys, as school-aged boys with internalizing symptoms are more likely to be referred to treatment than girls. ${ }^{18}$ However, Meltzer and colleagues ${ }^{15}$ suggest that "gender atypical" disorders may be more burdensome. There may also be differences by specific anxiety diagnosis (e.g., because gender norms emphasize the importance of relationships for girls, having a girl with social phobia could have more impact).

Drawing from the Duke Preschool Anxiety Study, a study of the epidemiology of preschool GAD, SAD, and SP in primary care, we sought to address the following questions: Do children's anxiety disorders have a perceived impact on family functioning? What is the relative impact of caring for a child with anxiety compared to other preschool disorders? Does perceived impact persist after accounting for the effects of comorbidity? We hypothesized that families of children with anxiety would report a significant negative impact on family functioning, even after accounting for the impact of other disorders, and that this impact would be similar to that of oppositional defiant disorder/conduct disorder (ODD/CD), attention-deficit hyperactivity disorder (ADHD), and depression. We were particularly interested in comparisons with ODD/CD and ADHD, given prior speculation that these disorders have a greater impact on the family than anxiety. ${ }^{4}$ Finally, does perceived impact of preschool anxiety on parent and family functioning vary according to the child's age and sex? Although these analyses were exploratory, we hypothesized no agebased differences for preschoolers, but that anxiety in boys would have a greater perceived impact.

\section{Method}

Study design and sample characteristics-Families in the Duke Preschool Anxiety Study were recruited from primary care clinics using a psychopathology screen-stratified 
design; a wide variety of children received care in these clinics, including those with private insurance, Medicaid, and those who were uninsured. First, children were screened for anxiety within the primary care population. Second, a subset of children was selected to participate in the in-home interview portion of the study, oversampling for those screening high for anxiety. Oversampling procedures provided an adequately representative sample of children with anxiety disorders to address project goals, with weighting procedures used to obtain unbiased estimates from the pediatric primary care population (for a review on oversampling techniques in epidemiology, see ${ }^{19}$ ).

Inclusion criteria for screening were (1) the child was 24-71-months-old, (2) attended the clinic during the screening period, and (3) a parent/legal guardian was present and consented to screening. Exclusion criteria were (1) lack of a parent/legal guardian with adequate fluency in English, (2) the child was known to have mental retardation (IQ < 70), autism, or other pervasive developmental disorders, (3) the child's sibling was already participating, (4) the child was not accompanied by a legal guardian who could provide consent, or (5) the child was considered by the provider to be too medically ill on the day of screening to participate. While the child and caregiver were in the examination room, the nurse asked if the caregiver was willing to speak with the research assistant about a research study. If willing, the caregiver provided written consent and completed the 10-item, narrow-band anxious/depressed subscale of the CBCL/1 $1 / 2-5^{20}$. "Screen high" scores were adjusted to select children scoring in the top 30\% of the clinic population (average score $=5.6$ ). The CBCL $/ 1 \frac{1}{2}-5$ was chosen because it is widely used as a screening tool and has been validated within a preschool population. ${ }^{21}$ Of the 4,520 2-5-year-olds attending the clinic during screening, $519(11.5 \%)$ were excluded from screening because they met the exclusion criteria noted above. Of the 4,001 eligible children, 522 (13\%) refused to participate, 46 $(1.1 \%)$ families missed contact, and 3,433 parents completed the screen $(85.8 \%$ of those eligible).

Of the families screened, 943 children (27.5\%) "screened high" for anxiety, all of whom were selected to participate in the in-home interview phase. Of the 2,490 children (72.5\%) who did not screen high, 189 (7.6\%) were randomly selected to participate in the in-home interview with the goal of providing an adequately-sized non-anxious comparison group. Of the 1,132 total children selected, $196(17.3 \%)$ refused and $19(1.7 \%)$ were excluded due to new information pertaining to the original exclusion criteria. A total of 917 parents (82.4\%) completed the in-home interview; the caregiver was given $\$ 75$ for completion of the inhome assessment. There were no significant differences by child age or sex between screen completers and non-completers, or those selected and those who completed the in-home interview. During the in-home interview, the Preschool Age Psychiatric Assessment (PAPA) and the Child and Adolescent Impact Assessment (CAIA) were administered; information was also collected on parent psychopathology, child symptoms and temperament, family functioning and service receipt.

\section{Measures}

Diagnostic assessment-Parents were interviewed using the PAPA, ${ }^{22}$ an interviewerbased diagnostic assessment for 2-5-year-olds. A wide range of DSM-IV diagnostic criteria relevant to younger children are assessed (e.g., anxiety, disruptive disorders, depression, ADHD), as well as all items in the DC:03-R. ${ }^{7}$ The interview is based on a set of required questions and probes, with discretionary follow-up probes to obtain adequate assessment of the presence and extent of child symptoms. When symptoms are reported, information is collected on the frequency, duration, and onset for a three-month primary period to establish whether criteria are met for DSM-IV diagnoses, as well as whether the child received treatment for these symptoms (i.e., referral to any professional agency (daycare, school, 
clinic, hospital, etc.)). All anxiety disorder diagnoses required the presence of both distress and impairment in functioning. Interviewers completed 1-2 weeks of classroom didactics and 1-2 weeks of practice in training for PAPA administration. A qualified PAPA trainer certified all interviewers prior to data collection, which were audiotaped for later quality control (see ${ }^{22}$ for further information on training). Diagnoses and symptom scales are generated using computerized algorithms written using SAS software. Demographic information was also collected via the PAPA. The test-retest and diagnostic reliability of the PAPA is comparable to similar diagnostic interviews with older children and adults, ${ }^{22,23}$ and provides symptom differentiation in accordance with DSM-IV nosology, suggesting similar construct validity (with respect to diagnostic specificity) as diagnostic interviews with older children. ${ }^{9}$

Perceived impact of child problems on the family-The CAIA ${ }^{24}$ was administered after the diagnostic interview to assess whether and how parents perceived that their child's problems affected family functioning. Parents reported on the impact of any problematic child behavior, including normative behavior (e.g., occasional tantrums, fear of the dark) for children without a disorder, and child symptomatology where relevant. The CAIA measures parent perception of 20-24 potential impacts of their child's problems (depending on the number of current and prior relationships), across four domains: 1) Economic, covering expenses, debts, and loss of income, 2) Relationships, including the impact on relationships with the parent's current or previous partner, other children, other family members, or friends, 3) Activities, including restrictions on personal or family social activities, and 4) Well-Being, including the parent's depression, fatigue, worries, irritability, and other aspects of mental or emotional health The presence of any perceived impact $(0=$ no negative impact, $1=$ impact on one or more domains) and the sum of the number of impacted domains (ranging 0-4) were used in the current analyses to assess the extent of impact. Although the CAIA has only been applied to school-aged children and adolescents to date (with high internal reliability $(a>.80)$ and adequate test-retest stability $(\mathrm{ICC}=.67)$ ), it was designed to assess broad domains of subjective and objective impact that child disorders might have across development. ${ }^{25}$

Data analysis-Because children with anxiety were oversampled, weighting procedures (with robust standard errors) were used to provide unbiased parameter estimates and confidence intervals for the relationship between variables in the pediatric primary care population. ${ }^{19}$ Sample weights were proportional to the inverse of each individual's probability of selection into the sample. Weighted logistic and Poisson regression models were estimated to examine the presence and degree of impact (number of impacted domains), respectively, for preschool anxiety compared to non-disordered children. To determine whether impact was driven by comorbidity, we then estimated anxiety effects after covarying the impact of ODD/CD, depression, and ADHD (using Type I sums of squares with other diagnoses entered first, and anxiety disorders last). Next, to examine the relative perceived impact of anxiety, separate independent $t$-tests were estimated comparing the impact of anxiety versus other disorders (for pairwise comparisons, children who met criteria for both disorders were excluded). Due to the large number of comparisons made across disorders, Bonferroni corrections were applied to all pairwise comparisons. Finally, weighted Poisson regression models were estimated to examine the family impact of anxiety by child age and sex, covarying relevant child and family factors.

Using Gpower software, ${ }^{26}$ power analyses assuming two-tailed tests with 0.80 power and a significance level of 0.05 were estimated to assess our ability to detect hypothesized effects. For the dichotomous impact outcome, $24 \%$ of families in the non-disordered sample reported family impact. The current study was sufficiently powered to detect an impact increase from $24 \%$ to $33 \%$. For continuous outcomes examining relative family impact 
across disorders, the current study was sufficiently powered to detect medium effect sizes (range, $d=0.50-0.66$ ). For Poisson models examining relative family impact, the current study was sufficiently powered to detect a means ratio of 1.4-1.6.

\section{Results}

The weighted prevalence of any of the three anxiety disorders was $19.44 \%$ (95\% CI 15.3423.52); Table 1 presents the rates of GAD, SAD, and SP, as well as rates by demographics. The weighted prevalence of depression, ODD/CD, and ADHD were $1.74 \%$ (95\% CI 1.222.26), $7.07 \%$ (95\% CI 4.58-9.56), and $4.28 \%$ (95\% CI 2.55-6.00), respectively. Of children with anxiety, the most common comorbidity was an additional anxiety diagnosis (30\% had at least one additional anxiety disorder). The most common comorbidities with non-anxiety disorders were ODD/CD (22\%), and ADHD (17\%). Only 5\% of children with anxiety received services for their symptoms (7\% of GAD, $5 \%$ of SAD, and $4 \%$ of SP). On average, parents of children with anxiety reported negative impact on one domain of family functioning ( $M=.97$ domains impacted); see Figure 1 for the number of impacted domains for non-disordered children across anxiety disorders, and for ODD/CD, ADHD, and depression.

\section{Presence and degree of perceived family impact for preschool anxiety}

Relative to children without a disorder, families of children with anxiety were 4.5 times more likely to report some impact (odds ratio (OR) 95\% CI 3.1-6.3, $p<.0001$ ), and the number of impacted domains for children with anxiety was 4.1 times greater than children without a disorder (risk ratio (RR) 95\% CI 3.3-5.1, $p<.0001$ ). Even after covarying the impact of other disorders (ODD/CD, depression, or ADHD), families of children with anxiety were 3.5 times more likely to report some family impact relative to those without a disorder (OR 95\% CI 2.4-5.3, $p<.0001$ ), and the number of impacted domains for children with anxiety was 3.3 times greater than children without a disorder (RR 95\% CI 2.6-4.3, $p$. 0001).

\section{Relative perceived family impact of preschool anxiety disorders}

Across all disorders, parents reported the greatest impact on their own well-being, and the least on economic burden. After the impact on their own well-being $(M=1.27,1.00$, and .80 for GAD, SAD, and SP, respectively) parents of children with GAD and SAD reported the most negative impact on their relationships $(M=.71, .64)$, whereas parents of children with SP reported the greatest impact on restrictions in their activities (e.g., social life, hobbies, etc.; $M=.40)$.

No significant differences emerged for perceived impact of GAD versus disruptive disorders or ADHD, $t(198)=1.46, p=.15$ and $t(185)=1.11, p=.20$, respectively. Depression had greater perceived impact than GAD $(t(165)=2.28, p<.05, d=.58)$. Specifically, GAD had less perceived impact on parental well-being than depression $t(165)=3.67, p<.001, d=.78$.

The perceived impact of SAD did not differ from that of ADHD $t(198)=1.53, p=.13$. SAD had less perceived impact on the family than disruptive disorders or depression $t(224)=1.93$, $p=.05, d=.29$ and $t(188)=3.85, p<.001, d=.88$, respectively. Specifically, SAD had less perceived impact on family activities than disruptive disorders $(t(224)=2.23, p<.05, d=.32)$; SAD had less perceived impact on family activities, relationships, and parental well-being than depression $(\mathrm{t}(21.7-188)=2.13-3.90, p<.05-.001, d=.60-.82)$.

Social phobia had significantly less perceived impact on the family than disruptive disorders $(t(189)=2.93, p<.01, d=.53)$, depression $(t(153)=3.49, p<.01, d=.68)$, or ADHD $(t(169)=2.82$, 
$p<.01, d=.48)$. Specifically, SP had less perceived impact on family activities, relationships, and parental well-being than disruptive disorders and depression $(\mathrm{t}(153-169)=2.28-2.61$, $p<.05-.01, d=.39-.44 ; \mathrm{t}(31-127)=2.16-2.65, p<.05-.01, d=.42-.56 ;(\mathrm{t}(44-162)=3.25-4.85$, $p<.01-.001, d=.49-1.03$, respectively). SP had less perceived impact on family activities and well-being than $\operatorname{ADHD}(t(168)=2.41, p<.01, d=.40$ and $t(97)=3.50, p<.001, d=.61)$.

\section{Perceived impact of child anxiety by sex, age, and comorbidity, covarying relevant child and family factors}

Model I in Table 2 presents the results of weighted Poisson regression analyses predicting perceived family impact for GAD, SAD, and SP. GAD and SAD significantly predicted impact (with an effect size of .41 and .45 , respectively); however, SP was unrelated when other diagnoses, child, and family factors were included in the model. Overall, marital status, child age, race, and enrollment in preschool or daycare also predicted impact, and there were significant main effects for disruptive disorders and depression. Specifically, married parents, families with older or Black children, children who did not meet criteria for disruptive disorders or depression, and children enrolled in preschool or daycare reported less impact.

Model II in Table 2 shows results from models exploring interactions between anxiety diagnosis and child age and sex; interaction terms were first tested in separate models, with significant interactions retained. The interaction between SP and child sex was significant in predicting impact on the family. For girls, the presence of SP was associated with greater perceived impact $(\beta=.63, p<.001)$; however, no difference was observed for boys $(\beta=-.26$, $p=.30$ ). The perceived impact of anxiety disorders did not differ based on child age.

\section{Discussion}

Our findings suggest that many young children experience distressing and impairing anxiety, with symptoms that have serious implications for family functioning. Contrary to the notion that anxiety disorders are less burdensome on families compared to disorders with higher rates of treatment (such as ADHD), our results indicate that the perceived impact of GAD and SAD are similar to that of attention problems. Comparisons with disruptive disorders were more mixed, with GAD and SAD showing similar levels of perceived impact with one exception: SAD does not appear to restrict family activities as much as disruptive behavior problems. Depression had a greater perceived impact on family functioning than anxiety, and social phobia had less impact than other disorders. Additionally, although comorbidity is present in early anxiety, the perceived impact of generalized and separation anxiety does not appear to be driven by this comorbidity; our findings suggest parents perceive anxiety disorders as having a unique, negative impact on the family.

In addition to the impairment early anxiety has on children's functioning and development, ${ }^{2,3}$ our results suggest that this impairment extends to parents' perception of family functioning, particularly their own well-being and adjustment; increased worries, depression, fatigue, and health problems were most commonly reported, followed by disrupted relationships and restricted personal or social activities. Similar to other disorders, having a child with anxiety may be an ongoing source of stress, undermining confidence in the parenting role and resulting in self-blame or shame. ${ }^{27}$ The stigma associated with child mental illness may reduce participation in social activities or relationships that could serve as a source of support, in addition to the competing demands of the child's illness. Because parents' adjustment, social isolation, and role restriction have important implications for children's long-term functioning, ${ }^{28}$ the perceived impact of anxiety is not only important in its own right, but also as a contributing factor to maintaining or increasing child problems over time. 
While the presence of additional disorders likely contributes to the cumulative family impact, our findings suggest that the perceived impact of anxiety is far from a mere reflection of comorbidity with disruptive behavior, depression, or ADHD. After accounting for comorbidity, GAD and SAD had unique perceived impact; the perceived impact of SP was qualified by characteristics of the child -- there was a significant impact on the family for girls with SP, whereas there was no impact for boys. In general, preschool boys have fewer social interactions than girls (e.g., boys have fewer play dates) ${ }^{29}$; with fewer opportunities to observe the child's difficulties, the unique impact of social phobia may be mitigated.

Despite evidence for significant impairment linked with preschool GAD and symptom differentiation from temperament and other anxiety disorders, ${ }^{30}$ there has been little research establishing the validity of this diagnosis in early childhood (particularly under the age of 3 ). With research suggesting less symptom differentiation and less short- and long-term stability in preschool GAD compared with other anxiety disorders, ${ }^{11-12,30}$ there is a need for further research on how to define and assess this disorder in young children. However, the unique perceived impact of GAD on family functioning adds to the growing body of work on the validity of this diagnosis for preschoolers, indicating both child and family impairment. Given suggestion of developmental variation in preschool GAD symptoms, ${ }^{13}$ it is of particular note that perceived impact did not vary according to the age of the child.

Although not central to the current investigation, the impact reported by parents for preschool depression is also notable. Evidence suggests preschool depression impairs multiple domains and contexts, with symptoms of guilt and extreme fatigue differentiating depression from other disorders. ${ }^{31}$ It is possible that the high impact noted is due to the unique symptoms of depression, or the relatively recent recognition of this disorder in young children. Alternatively, parents of children with depression may be at risk for stigmatization, heightening family impact.

A number of study limitations should be noted. First, due to the cross-sectional and correlational nature of these analyses, it is impossible to tease apart the direction of effects between reported impact and the child's disorder, or eliminate the possibility that linkages found result from an unmeasured factor. For example, it may be that parents with disorders are both more likely to have a child with anxiety and to perceive negative impact, or that associations between child anxiety and parent's perception of impact may be due to a shared propensity to distress or environmental factors. Additionally, one parent was the sole reporter of diagnostic and impact data. Although parental informants may be particularly important for young children, inclusion of observations or child interviews would provide a multi-method assessment that could reduce the effects of shared method variance. Because of the design of the Duke Preschool Anxiety Study, we were unable to adequately address the impact of specific phobias, obsessive compulsive, and post-traumatic stress disorders. Finally, the prevalence rates and results generalize to pediatric clinics, as the sample was drawn from this setting. While pediatric primary care is an important avenue for identification and treatment of early disorders, ${ }^{4}$ whether the prevalence and impact of preschool anxiety is similar in the general population is unclear.

Despite these limitations, this study advances our understanding of early anxiety in a number of ways. Drawing from a large, primary care pediatric sample and oversampling for children at risk for anxiety, we were able to identify and examine the perceived impact of specific anxiety disorders and comorbidity, likely resulting in a more representative sample of children with anxiety than reliance on clinical samples. Although there are limitations in current diagnostic assessments of preschool psychopathology, ${ }^{5}$ our use of the PAPA provided greater confidence in our measurement of anxiety diagnoses, as it is the only 
diagnostic interview reporting acceptable levels of test-retest reliability and validity for preschool-aged children. ${ }^{19}$ In addition, this is the first empirical examination of the perceived impact of preschool anxiety, as well as the relative impact of early childhood disorders.

In terms of understanding the lack of treatment for young children with anxiety, ${ }^{4}$ our findings suggest there are other factors aside from family impact that should be explored; generalized and separation anxiety have as much perceived impact as other disorders with higher rates of treatment (e.g., ADHD). Only 5\% of preschoolers with anxiety in our sample received treatment for their symptoms; there is clearly a significant gap between the prevalence of preschool anxiety and its treatment. Lack of awareness of the prevalence of early anxiety or tools for identifying these disorders may be one reason for the relative lack of treatment. The importance of educating parents and providers on the symptoms and assessment of early disorders has been repeatedly noted, ${ }^{32}$ and this may be particularly important for childhood anxiety, given its relative under-identification. The limited number of evidence-based treatments for early anxiety may also be a critical barrier, because parents and providers may be less likely to pursue services in the absence of knowledge about effective interventions.

Our findings also underscore the importance of including the family in the treatment of early anxiety. Emerging evidence suggests cognitive-behavior based parenting interventions are effective in treating anxiety in 3-to-9-year olds, ${ }^{33}$ and parent-education prevention programs have been shown to reduce anxiety for preschoolers with anxiety disorders. ${ }^{30}$ Because of the perceived impact of child anxiety on parental adjustment, family-based treatment programs that teach parents how to manage their own negative emotions, as well as how help their children do so, may be particularly beneficial in reducing both child and family impairment. Given the prevalence and impact of preschool anxiety, this is clearly an important area for further work.

\section{Acknowledgments}

This research was supported by a grant from National Institute of Mental Health (RO1-MH-075766). Additional support was provided through a grant from National Institute of Child Health and Human Development (T32HD-007376; NRTG).

\section{References}

1. Costello, EJ.; Egger, HL.; Angold, A. The developmental epidemiology of anxiety disorders. In: Ollendick, T.; March, J., editors. Phobic and anxiety disorders in children and adolescents: A clinician's guide to effective psychosocial and pharmacological interventions. NY: Oxford University Press; 2004. p. 61-91.

2. Egger, HL.; Angold, A. Anxiety disorders. In: Luby, J., editor. Preschool Mental Health: A Guide for Practitioners. New York: Guilford; 2006. p. 137-164.

3. Sterba S, Prinstein M, Cox M. Trajectories of internalizing problems across childhood: Heterogeneity, external validity, and gender differences. Dev Psychopathol. 2007; 19:345-366. [PubMed: 17459174]

4. Chavira DA, Stein MB, Bailey K, Stein MT. Child anxiety in primary care: Prevalent but untreated. Depress Anxiety. 2004; 20:155-164. [PubMed: 15643639]

5. Egger H, Emde RN. Developmentally sensitive diagnostic criteria for mental health disorders in early childhood. Am Psychol. 2011; 66:95-106. [PubMed: 21142337]

6. Scheeringa M. Research diagnostic criteria for infants and preschool children: The process and empirical support. J Am Acad Child Adolesc Psychiatry. 2003; 42:1504-1512. [PubMed: 14627886] 
7. Zero to Three. Diagnostic Classification: 0-3R: Diagnostic Classification of Mental Health and Developmental Disorders of Infancy and Early Childhood: Revised Edition. Washington, DC: Zero To Three Press; 2005.

8. Carter AS, Briggs-Gowan MJ, Davis NO. Assessment of young children's social-emotional development and psychopathology: Recent advances and recommendations for practice. J Child Psychol Psychiatry. 2004; 45:109-134. [PubMed: 14959805]

9. Eley TC, Bolton D, O'Connor TG, Perrin S, Smith P, Plomin R. A twin study of anxiety-related behaviors in pre-school children. J Child Psychol Psychiatry. 2003; 44:945-960. [PubMed: 14531577]

10. Pahl K, Barrett PM, Gullo MJ. Examining potential risk factors for anxiety in early childhood. J Anx Disorders. 2012; 26:311-320.

11. Bufferd SJ, Dougherty LR, Carlson GA, Rose S, Klein DN. Psychiatric disorders in preschoolers: Continuity from ages 3 to 6. Am J Psychiatry. 2012; 169:1157-1164. [PubMed: 23128922]

12. Sterba S, Egger HL, Angold A. Diagnostic specificity and nonspecificity in the dimensions of preschool psychopathology. J Child Psychol Psychiatry. 2007; 48:1005-1013. [PubMed: 17915001]

13. Mian ND, Godoy L, Briggs-Gowan MJ, Carter AS. Patterns of anxiety symptoms in toddlers and preschool-age children: Evidence of early differentiation. J Anxiety Disord. 2012; 26:102-110. [PubMed: 22018968]

14. Angold A, Messer SC, Stangl D, Farmer EM, Costello EJ, Burns BJ. Perceived parental burden and service use for child and adolescent psychiatric disorders. J Am Pub Health. 1998; 88:75-80.

15. Meltzer H, Ford T, Goodman R, Vostanis P. The burden of caring for children with emotional or conduct disorders. Intl J Fam Med. 2011:1-8.

16. Chavira DA, Garland A, Yeh M, McCabe K, Hough RL. Child Anxiety Disorders in Public Systems of Care: Comorbidity and Service Utilization. J Behavl Health Ser R. 2009; 36:492-504.

17. Egger, HL.; Burns, BJ. Anxiety disorders and access to mental health services. In: Ollendick, T.; March, J., editors. Phobic and anxiety disorders in children and adolescents. NY: Oxford; 2004. p. 530-549.

18. Laitinen-Krispijn S, DerEnde J, Wierdsma A, Verhulst F. Predicting Adolescent Mental Health Service Use in a Prospective Record-Linkage Study. J Am Acad Child Adolesc Psychiatry. 1999; 38:1073-1080. [PubMed: 10504805]

19. Kalsbeek W, Heiss G. Building bridges between populations and samples in epidemiological studies. Annu Rev Publ Health. 2000; 21:147-169.

20. Achenbach, TM.; Rescorla, LA. ASEBA Preschool Forms and Profiles: An Integrated System of Multi-informant Assessment. ASEBA; 2000.

21. Koot HM, Oord JCG, Verhulst FC, Boomsma DI. Behavioral and emotional problems in young preschoolers: Cross-cultural testing of the validity of the Child Behavior Checklist/2-3. J Abnorm Child Psych. 1997; 25:183-196.

22. Egger, HL.; Angold, A. The Preschool Age Psychiatric Assessment (PAPA): A structured parent interview for diagnosing psychiatric disorders in preschool children. In: DelCarmen-Wiggins, R.; Carter, A., editors. Handbook of infant, toddler, and preschool mental health assessment. NY: Oxford; 2004. p. 223-243.

23. Egger HL, Erkanli A, Keeler G, Potts E, Walter BK, Angold A. Test-retest reliability of the Preschool Age Psychiatric Assessment. J Am Acad Child Adolesc Psychiatry. 2006; 45:538-549. [PubMed: 16601400]

24. Angold, A.; Patrick, M.; Burns, BJ.; Costello, EJ. The Child and Adolescent Impact Assessment (CAIA: Version 3.0). Department of Psychiatry and Behavioral Sciences; Durham, NC: Duke University Medical Center; 2008.

25. Messer SC, Angold A, Costello EJ, Burns BJ. The child and adolescent burden assessment (CABA): Measuring the family impact of emotional and behavioral problems. Int J Method Psych. 1998; 6:261-284.

26. Faul F, Erdfelder E, Lang A, Buchner A. G*Power 3: A flexible statistical power analysis program for the social, behavioral, and biomedical sciences. Behav Res Method. 2007; 39:175-191. 
27. Hinshaw SP. The stigmatization of mental illness in children and parents: Developmental issues, family concerns, and research needs. J Child Psychol Psychiatry. 2005; 46:714-734. [PubMed: 15972067]

28. Crnic, K.; Low, C. Everyday stresses and parenting. In: Bornstein, M., editor. Handbook of Parenting:Volume 5, Practical Issues and Parenting. Mahwah, NJ: Erlbaum; 2002. p. 243-267.

29. Gottman JM. How children become friends. Monogr Soc Res Child. 1983; 48:1-86.

30. Egger HL, Angold A. Common emotional and behavioral disorders in preschool children: Presentation, nosology, and epidemiology. J Child Psychol Psychiatry. 2006; 47:313-337. [PubMed: 16492262]

31. Luby J, Belden A, Pautsch J, Si X, Spitznagel E. The clinical significance of preschool depression: Impairment in functioning and clinical markers of the disorder. J of Affect Disorders. 2009; 112:111-119. [PubMed: 18486234]

32. Cartwright-Hatton S, McNally D, Field A, et al. A new parenting-based group intervention for young anxious children: Results of a randomized controlled trial. J Am Acad Child Adolesc Psychiatry. 2011; 50:242-251. [PubMed: 21334564]

33. Rapee RM, Kennedy S, Ingram M, Edwards S, Sweeney L. Prevention and early intervention of anxiety disorders in inhibited preschool children. J Consult Clin Psych. 2005; 73:488-497. 


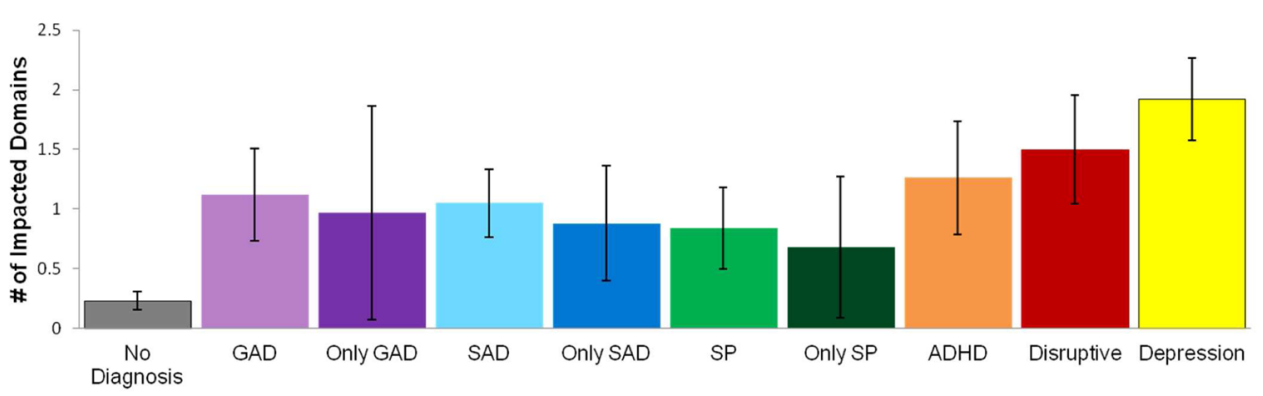

Figure 1.

Weighted mean number of impacted domains by disorder (95\% Confidence Intervals) $(n=$ 917). Note: $A D H D=$ attention-deficit/hyperactivity disorder; GAD = Generalized Anxiety Disorder; ODD/CD = Oppositional Defiant Disorder/Conduct Disorder; SAD = Separation Anxiety Disorder; SP = social phobia. 


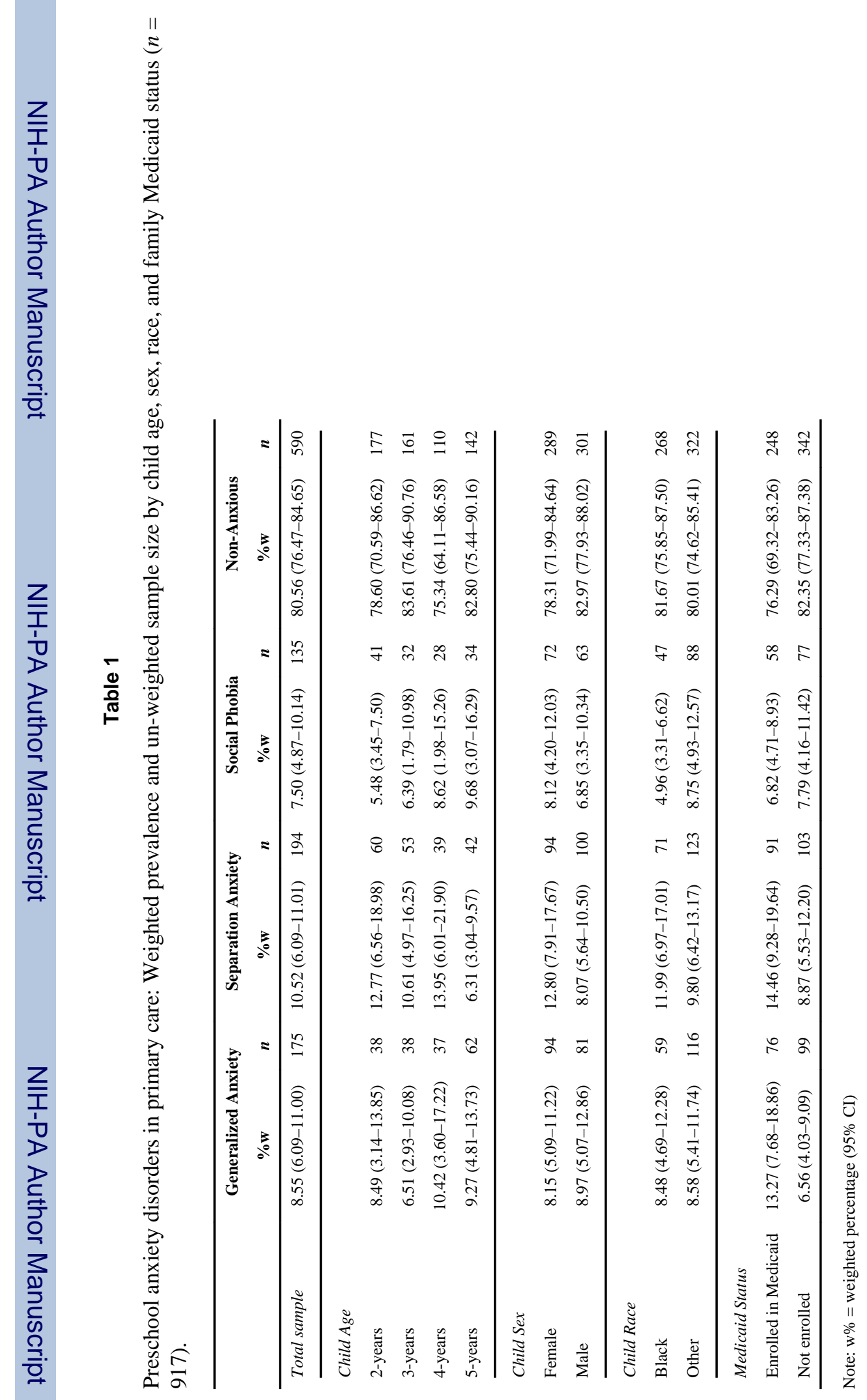


Table 2

Weighted Poisson regression analyses predicting perceived impact of child problems: Main effects and interactions with child sex $(n=917)$.

\begin{tabular}{lcccc}
\hline & \multicolumn{2}{c}{ Model I } & \multicolumn{2}{c}{ Model II } \\
\hline & OR & $\mathbf{9 5 \%}$ CI & OR & $\mathbf{( 9 5 \% ~ C I )}$ \\
\hline Child and Family Factors & & & & \\
Child age & $.91^{*}$ & $.84-.99$ & $.91^{*}$ & $.84-.99$ \\
Child sex $a$ & 1.17 & $.95-1.44$ & $1.34^{*}$ & $1.07-1.68$ \\
Child race $b$ & $.43^{* * *}$ & $.32-.57$ & $.44^{* * *}$ & $.33-.58$ \\
Daycare/Preschool $c$ & $.74^{*}$ & $.58-.95$ & $.73^{*}$ & $.57-.93$ \\
Medicaid status ${ }^{*}$ & .97 & $.72-1.31$ & .98 & $.73-1.33$ \\
Parent marital status ${ }^{e}$ & $.69^{*}$ & $.52-.92$ & $.73^{*}$ & $.54-.97$ \\
Disorder & & & & \\
Disruptive (ODD/CD) & $2.65^{* * *}$ & $2.02-3.47$ & $2.64^{* * *}$ & $2.01-3.46$ \\
Depression & $1.70^{*}$ & $1.11-2.62$ & $1.68^{*}$ & $1.10-2.58$ \\
ADHD & 1.33 & $.94-1.89$ & $1.43^{*}$ & $1.00-2.03$ \\
GAD & $1.37^{*}$ & $1.02-1.83$ & $1.40^{*}$ & $1.04-1.87$ \\
SAD & $1.84^{* * *}$ & $1.41-2.39$ & $1.86^{* *}$ & $1.42-2.43$ \\
SP & 1.29 & $.95-1.76$ & $1.87^{* *}$ & $1.29-2.72$ \\
Interactions & & & & $.41^{* *}$ \\
SP x Child sex & & & $.23-.75$ \\
\hline
\end{tabular}

Note: Interaction terms were tested in separate models; only significant interactions were retained for entry in final model;

$*$

$\mathrm{p} \leq 05$,

**

$\mathrm{p} \leq 01$,

$* * * \mathrm{p} \leq 001 ;$

${ }^{a}$ Sex: $1=$ Male, $0=$ Female;

${ }^{b}$ Race: $1=$ Black, $2=$ Other;

${ }^{c}$ Daycare/School: 1=Enrolled in Daycare or School for at least 1 day a week for past 3 months, 0=Not Enrolled;

${ }^{d}$ Medicaid Status: 1=Enrolled in Medicaid/CHIPS, 0=Not Enrolled;

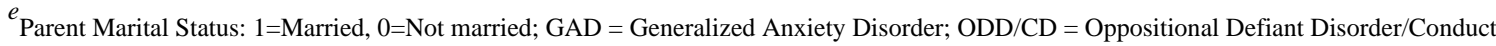
Disorder; SAD = Separation Anxiety Disorder; SP = social phobia. 